\title{
Is increased sensitivity to punishment a common characteristic of attention deficit/hyperactivity disorder? An experimental study of response allocation in Japanese children
}

\author{
Emi Furukawa ${ }^{1}$ [ $\cdot$ Brent Alsop $^{2} \cdot$ Shizuka Shimabukuro $^{1} \cdot$ Gail Tripp ${ }^{1}$
}

Received: 4 January 2019 / Accepted: 7 May 2019 / Published online: 16 May 2019

(c) The Author(s) 2019

\begin{abstract}
Research on motivational processes in attention deficit/hyperactivity disorder (ADHD) focuses on reward. Studies with punishment are limited and findings mixed. This study evaluated the effects of punishment on response allocation in Japanese children with and without ADHD. Thirty-four children meeting DSM-IV criteria for ADHD and 59 typically developing control-group children completed an operant task in which they choose between playing two simultaneously available games. Reward was arranged symmetrically across the games under concurrent variable interval schedules. Asymmetric punishment schedules were superimposed with responses on one game punished four times as often as responses on the other. Children with ADHD showed greater behavioral sensitivity to punishment than controls. They allocated significantly more responses to the less frequently punished alternative and were more likely to play this game on consecutive trials and responded more slowly to the more punished game. Control group children allocated their responses evenly across games. Punishment exerted greater control over the behavior of Japanese children with ADHD than controls, similar to findings with children from Western countries, suggesting this is a common characteristic of the disorder. The behavior of typically developing Japanese children, while demonstrating awareness of punishment, was not controlled by the frequency of its occurrence.
\end{abstract}

Keywords ADHD $\cdot$ Motivation $\cdot$ Punishment $\cdot$ Response allocation $\cdot$ Japanese

\section{Introduction}

Motivational accounts of attention deficit/hyperactivity disorder (ADHD) propose that altered processing of reinforcement contingencies contributes to the disorder's symptoms (Luman et al. 2010). These hypotheses emphasize sensitivity to positive reinforcement, and this is reflected in the research literature (e.g., Luman et al. 2005; Alsop et al. 2016). Despite recognition that behavior is affected by both positive

Electronic supplementary material The online version of this article (https://doi.org/10.1007/s12402-019-00307-6) contains supplementary material, which is available to authorized users.

Emi Furukawa

furukawa@oist.jp

1 Human Developmental Neurobiology Unit, Okinawa Institute of Science and Technology Graduate University, 1919-1 Tancha, Onna, Okinawa 904-0495, Japan

2 Department of Psychology, University of Otago, Dunedin, New Zealand and negative consequences, the sensitivity of children with ADHD to punishment has received limited theoretical and experimental attention.

In daily life, ADHD is associated with inappropriate risktaking behavior in children (Badger et al. 2008; Garzon et al. 2008), adolescents and adults (Flory et al. 2006; Thompson et al. 2007; Lee et al. 2011), possibly reflecting reduced sensitivity to negative outcomes. In experimental settings, children and adolescents with ADHD have sometimes been shown to make more risky choices during gambling tasks with a penalty component (see Groen et al. 2013 for a review), leading to suggestions that children with ADHD are less sensitive to the effects of punishment than their typically developing peers. However, these findings could also be explained by suboptimal decision making. Pollak et al. (2016) have shown equalizing the expected value of risky and safe choices leads to a similar number of risky choices in those with ADHD and controls. Using a sequential risky decision-making task (Balloon Emotional Learning Task), Humphreys et al. (2018) also found ADHD status was not associated with increased risk taking. Rather they suggest 
learning during decision making may be more difficult for children with ADHD.

Mild punishment (response cost) has been shown to reduce off-task or maintain on-task behavior in children with ADHD (Worland 1976; Rapport and Bailey 1982; Rosén et al. 1984) and to enhance their performance on cognitive tasks (e.g., Crone et al. 2003; van Meel et al. 2005; Drechsler et al. 2010), with some studies reporting improved performance in those with ADHD only (e.g., Iaboni et al. 1995; Carlson et al. 2000), indicating intact or possibly heightened behavioral sensitivity to punishment. This interpretation is consistent with the recent findings of Furukawa et al. (2017). Using a concurrent schedules procedure, they demonstrated that punishment exerts greater control over the response allocation of children with ADHD with increased time on task, i.e., children with ADHD are more sensitive to the cumulative effects of punishment than typically developing children.

While this recent study by Furukawa et al. (2017) argues that children with ADHD are more, rather than less, sensitive to the effects of punishment, additional research is needed to clarify the nature of altered sensitivity to punishment in children with ADHD. This should include research with non-Western populations, using the same or similar experimental paradigms to facilitate cross-cultural comparisons. Such studies are critical in determining if the link between altered motivational processes and ADHD generalizes across cultures. Sample diversity becomes increasingly important as Western-styled behavior management programs, relying on the use of reward and negative consequences to manage children's behavior, are progressively adopted into nonWestern cultures (Thompson et al. 2017). We identified only one study assessing punishment sensitivity in children with ADHD from a non-Western culture. Masunami et al. (2009) reported Japanese children with ADHD attended less to punishment than controls on a variant of the Iowa Gambling Task.

In this study, we evaluate the sensitivity of Japanese children with and without ADHD to punishment using the operant task described by Furukawa et al. (2017). The children completed a computer task in which they chose between playing two simultaneously available games (response alternatives). The two games arranged equal rates of reward using concurrent variable interval (VI/VI) schedules, over which punishment schedules were superimposed such that responses on one game were punished four times as often as responses on the other. The asymmetric punishment schedule, superimposed on the symmetric reinforcement schedule, should bias responding toward the less punished response alternative as demonstrated previously (Critchfield et al. 2003; Rasmussen and Newland 2008; Furukawa et al. 2017). The size of the bias provides a quantitative measure of the degree to which punishment exerts control over behavior.
Any group differences in behavioral sensitivity to punishment will appear as differences in this response bias.

If symptoms of ADHD are mediated by altered sensitivity to motivationally significant events, then children with the disorder would be expected to respond similarly to punishment, irrespective of their cultural background. Thus, we predict Japanese children with ADHD will show increased sensitivity to the cumulative effects of punishment as previously shown in American and New Zealand children with ADHD (Furukawa et al. 2017). If the children with ADHD allocate more of their responses to the less punished alternative, they will require additional trials to receive rewards, as these are arranged equally across the two games (Furukawa et al. 2017). Immediate effects of punishment might be reflected in slowed response times following a loss and a shift away from the punished game (Furukawa et al. 2017).

The sensitivity of typically developing Japanese children to punishment is more difficult to predict. While the neurobiology underlying responses to positive reinforcement (Wickens et al. 2003; Pan et al. 2005), and to a lesser extent punishment (Seymour et al. 2007; Jean-Richard-Dit-Bressel et al. 2018), are well established, there is some evidence that responsiveness to contingencies is environmentally mediated (Rothbart and Ahadi 2000; Kennedy et al. 2016). In Japan where humility is highly valued, the use of self-criticism and negative feedback to improve oneself and others is more normative (Kitayama et al. 1997; Heine et al. 1999). It is not clear if increased prior exposure to negative feedback will lead to increased behavioral sensitivity to punishment among typically developing Japanese participants, or if they will have habituated to its effects (Rothbaum et al. 2000).

\section{Method}

Ethical approval for the study was obtained from the Human Subjects Research Review Committee at the Okinawa Institute of Science and Technology (OIST) Graduate University, Japan. Participating parents, teachers, and children were volunteers and provided written consent.

\section{Participants}

Data from 93 children aged between 6 and 12 years, 34 meeting DSM-IV diagnostic criteria for ADHD (85\% boys) and 59 control group children (49\% boys) living in Okinawa, Japan, are included in the study. The majority of children lived in a two-parent household, and the household income and number of siblings living together were similar for the ADHD and control groups (see Supplemental Table 1). Within the ADHD group, 16 children met the diagnostic criteria for inattentive and 18 for combined type ADHD. 
Table 1 Participant characteristics for the ADHD and control groups

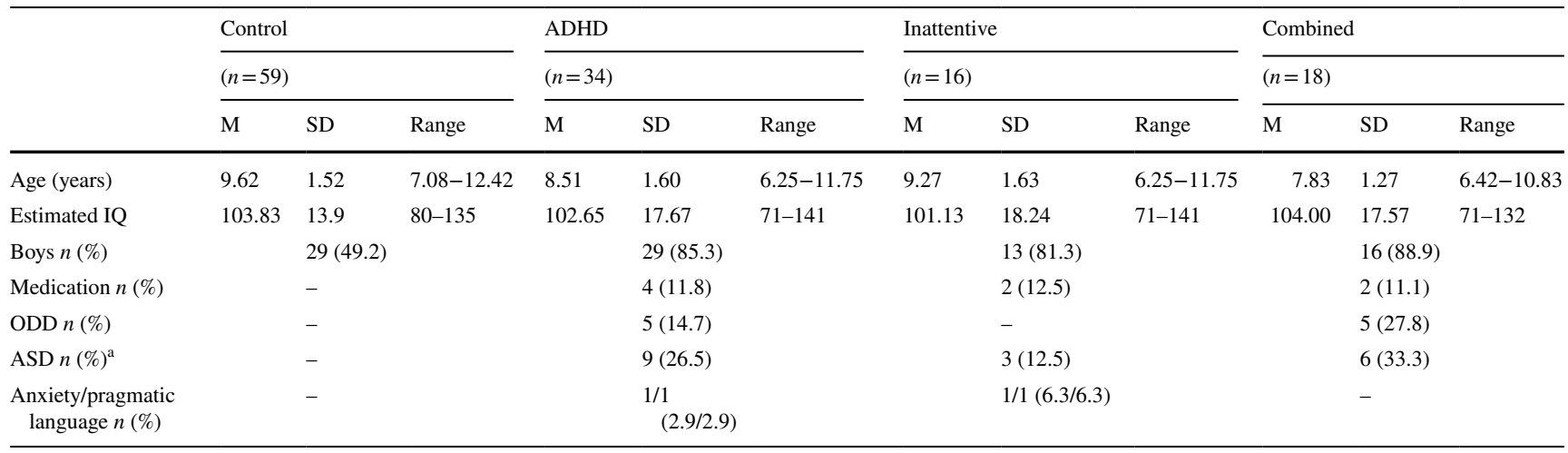

${ }^{a}$ Given that DSM-5 allows comorbid diagnosis of ASD with ADHD, children demonstrating symptoms of DSM-IV Asperger disorder were included in the study if they satisfied other inclusion criteria

Inclusion criteria were an estimated IQ of at least 70, normal or corrected vision, no past or current head injury, neurological disorder or psychosis, and native Japanese speakers. Comorbid conditions were allowed if the above inclusion criteria were met (Table 1). Children in the ADHD group were recruited through a University ADHD Research Center. Local schools, clinics, and parent support groups were informed about center's research. Interested parents contacted the center for additional information and participated in a telephone screening interview. Those reporting three or more symptoms of ADHD in their child were invited to participate in the center's research which included multimethod, multi-informant research diagnostic assessments for ADHD. Assessments were carried out by a research team including a US-licensed, bilingual (Japanese and English) clinical psychologist (EF), and Japanese-speaking clinicians with advanced counseling or other relevant degrees, all experienced in working with children with ADHD.

Assessments took place over three days. On the first day, clinical and semi-structured diagnostic interviews [K-SADSPL Disruptive Behavior Disorder sections (Miyawaki et al. 2003)] were conducted with the parents (typically the mother). During the next 2 days, the child completed tests of cognitive and academic functioning, a child interview, other tests necessary for differential diagnosis and experimental tasks, including the current paradigm. The parent interview, parent and teacher completed rating scales for ADHD symptoms [SNAP (Inoue et al. 2014)] and observations of the child's behavior during the assessment were used to make best estimate research diagnoses of ADHD. Parent and teacher completed broadband rating scales [CBCL/ TRF (Itani et al. 2001; Kawauchi et al. 2011)] screened for other behavioral and emotional problems (Supplemental Table 1). A background questionnaire, developed by the research team, inquired children's past and current medical conditions, medication intake, pre-natal, and post-natal development, academic, social and daily functioning as well as demographic information. Cognitive functioning was assessed with the WISC-III (Wechsler and Japanese WISCIII Publication Committee 1998) and WISC-IV (Wechsler and Japanese WISC-IV Publication Committee 2010).

For inclusion in the ADHD group, children were required to display a minimum of six symptoms of inattention and/ or hyperactivity/impulsivity in at least one setting, evidence of symptoms in a second setting, and functional impairment from symptoms, based on parent K-SADS interview summary scores, together with other available data (parent and teacher SNAP ratings and behavioral observation during testing). ${ }^{1}$ Four children were prescribed stimulant medication (Concerta), which was discontinued for at least $24 \mathrm{~h}$ prior to study participation. One of these children was concurrently prescribed a non-stimulant medication (Strattera), the last intake was the evening before the assessment. Sixteen children had at least one comorbid condition, including five with oppositional defiant disorder (ODD), and nine with a past diagnosis of Autistic, Asperger's or other pervasive developmental disorder (PDD) and demonstrating behavioral characteristics consistent with Asperger's disorder at the time of the assessment, without significant cognitive or language impairments. The high rate of comorbid PDD is consistent with the prevalence rate observed in psychiatry practices in Japan (Yoshida and Uchiyama 2004; Takanashi et al. 2014) and may reflect families of children with more severe impairments seeking evaluation. None of the children met criteria for conduct disorder (CD).

Children in the control group were recruited through invitation letters sent home to parents through public schools.

\footnotetext{
${ }^{1}$ In three cases, the mother's report during the K-SADS interview indicated five symptoms of inattention or hyperactivity/impulsivity. As more than six symptoms were indicated by another rater on the SNAP (teacher or father, symptoms not summed across informants), which concurred with behavioral observations during testing, these cases were judged to meet the DSM-IV diagnostic criteria for ADHD.
} 
These children completed an abbreviated IQ assessment (WISC-III Vocabulary/Block Design). Their parents and teachers completed the behavior rating scales, which ruled out the presence of ADHD or other behavioral or emotional disorders. Those demonstrating fewer than three symptoms of either inattention or hyperactivity/impulsivity were included. Parent and teacher ratings for these children did not yield any scores in the clinical range on any of the problem behavior scales (CBCL/TRF). Parental questionnaire reports of medical and developmental history were reviewed for other inclusion criteria.

Table 1 presents the sample characteristics of the ADHD and control groups. No significant difference was identified for estimated IQ scores. The mean age of the control group was significantly older than the ADHD group $(t(91)=3.33$, $p<.01)$, and the ADHD group included a higher proportion of boys $\left(x^{2}(1, N=93)=12.00, p<.01\right)$.

\section{Experimental task}

The children sat approximately $400 \mathrm{~mm}$ from a flat-screen monitor with a mouse. The task (Supplemental Figure 1) began with the computer presenting the instructions that were read aloud by the experimenter. Two $2 \times 2$ grids of cartoon characters were presented on the screen, one on the left (Game 1) and one on the right (Game 2), with a center window displaying accumulated points. All children began with a balance of 20 points. They were told they could switch between playing the two games as often as they liked and they should win lots of points to get a prize after the task ended. A mouse click on a button below each grid made the characters in the selected game spin for $3000 \mathrm{~ms}$. Whenever the four characters matched (reward trial), 10 points were added to their total, a randomly selected animated cartoon was displayed for 2500-3000 ms and a congratulatory "tada!" sound played. Whenever four sad-faced characters appeared together (punishment trial), 5 points were taken away from the child's total and a laughing sound "ha ha ha!" played for $5000 \mathrm{~ms}$. A mismatch of characters had no associated outcome (non-consequential trial). Once a choice was made, the response buttons were disabled until the trial was complete, including outcome delivery. The task continued until the child accumulated 400 points or completed 300 trials, whichever occurred first. All children completed at least 200 trials and received a small prize. Sessions typically lasted 30-35 min.

Rewards became available every $10 \mathrm{~s}$ on average arranged equally across the two games (VI/VI). Such concurrent variable interval schedules usually produce near equal allocation of behavior across the two response alternatives (Baum 1981; Borrero et al. 2010), including in children with ADHD (Taylor et al. 2010). The following constraints were programed: for each successive set of 12 rewards, each game delivered 6 rewards, randomized within each set; thus the longer a child played and won points on one game, the greater the likelihood the other game was scheduled for the next available reward. This ensured each child obtained the same arranged distribution of rewards in earlier and later trials and discouraged playing on one game exclusively. For each child, an equal percentage of trials were rewarded across the two alternatives; however, the percentages of trials rewarded differed between participants depending on how they allocated their responses. Rewards were never delivered on the first trial after switching games to avoid adventitious reinforcement of switching behavior (Stubbs and Pliskoff 1969), while the VI/VI reinforcement schedule encouraged the child to explore both games.

Punishments were arranged asymmetrically across the two games and were superimposed over the reward schedules. Each response on Game 1 had a $16 \%$ chance of being punished, with a $4 \%$ chance for Game 2 . The rates of reward and punishment were chosen, so children should, overall, win more points than they lose. These schedules were maintained throughout the task.

\section{Data collection and analyses}

On each trial, the computer recorded the game chosen, the outcome (win, loss or no outcome), and response time (recorded from the time the response buttons became available, after outcome presentation, until a response was made).

Response bias $(\log 10 b)$ was calculated by the following equation:

$\log 10 b=\log 10 \frac{\text { responses to Game } 2 \text { (less punished) }}{\text { responses to Game } 1 \text { (more punished) }}$

Scores above zero indicate a systematic preference for the less frequently punished response alternative.

Response bias, number of each outcome type, and median response times were calculated for each block of 100 trials. Mean scores were compared between the ADHD and control group for the first and second blocks of 100 trials, as all participants completed at least 200 trials before accumulating 400 points.

Instances of playing the same game for two consecutive trials (stays) were counted across blocks for each participant. The proportion of "stays" (number stay trials/total trials) were calculated separately for Game 1 and Game 2. These proportions were subjected to logit transformations; i.e., log 10 (proportion/( 1 - proportion)), group comparisons were made over the first 200 trials.

Counts were also made for stays following each trial type and converted to proportions for reward, punishment, and non-consequential trials (stay trials of one trial type/ total trials of that type). Group means were obtained for the 
Table 2 Means and standard errors for the bias score, proportion of stays, median response time, and performance (number of each outcome type and points accumulated) for each block of 100 trials for the ADHD and control groups

\begin{tabular}{|c|c|c|c|}
\hline \multirow[t]{2}{*}{ Group/trial block } & \multicolumn{2}{|l|}{$M(\mathrm{SE})$} & Third (trial 201-300) \\
\hline & \multicolumn{2}{|c|}{ (ADHD $n=34$, control $n=59$ ) } & (ADHD $n=31$, control $n=37$ ) \\
\hline \multicolumn{4}{|c|}{ Bias for less punished $(\log 10 b)$} \\
\hline ADHD & $.13(.04)$ & $.22(.06)$ & $.20(.07)$ \\
\hline Control & $.04(.03)$ & $.07(.04)$ & $.14(.06)$ \\
\hline \multicolumn{4}{|l|}{ Proportion of stays } \\
\hline ADHD & $.59(.03)$ & $.68(.03)$ & $.70(.04)$ \\
\hline Control & $.48(.02)$ & $.53(.03)$ & $.55(.04)$ \\
\hline \multicolumn{4}{|l|}{ Response time (s) } \\
\hline ADHD & $1.51(.10)$ & $1.27(.11)$ & $1.09(.07)$ \\
\hline Control & $1.88(.08)$ & $1.46(.08)$ & $1.09(.07)$ \\
\hline \multicolumn{4}{|l|}{ Wins (counts) } \\
\hline ADHD & $11.91(.81)$ & $11.99(.97)$ & $12.71(.90)$ \\
\hline Control & $13.85(.60)$ & $15.08(.71)$ & $12.73(.81)$ \\
\hline \multicolumn{4}{|l|}{ Losses (counts) } \\
\hline ADHD & $9.11(.61)$ & $8.24(.58)$ & $7.76(.67)$ \\
\hline Control & $8.36(.45)$ & $8.68(.43)$ & $8.23(.60)$ \\
\hline \multicolumn{4}{|c|}{ No outcome (counts) } \\
\hline ADHD & $78.98(.94)$ & $79.78(1.24)$ & $79.53(1.31)$ \\
\hline Control & $77.79(.69)$ & $76.25(.91)$ & $79.04(1.19)$ \\
\hline \multicolumn{4}{|c|}{ Points earned $(=$ wins $* 10-$ losses $* 5)$} \\
\hline ADHD & $73.56(9.11)$ & $78.67(9.37)$ & $88.33(8.19)$ \\
\hline Control & $96.68(6.70)$ & $107.38(6.89)$ & $86.13(7.43)$ \\
\hline
\end{tabular}

Adjusted for age and gender, 110.57 (in months) and 1.38 (1=male, $2=$ female) for the first and second block, 109.59 (in months) and 1.31 $(1=$ male, $2=$ female $)$ for the third block, respectively. Statistical analyses were conducted for the first and second blocks

logit-transformed values of these proportions across blocks. The proportion of stays were compared for punishments versus no outcomes, the proportion of stays following rewards was examined separately, over the first 200 trials. Trial types were separated this way as the first response after switching games was never rewarded, increasing the expected proportion of stays after non-rewarded trials compared to rewarded trials. Finally, median response times were calculated for each game and following each trial type for comparison within the control and ADHD groups over the first 200 trials.

Mixed ANOVA using SPSS GLM compared the performance of the ADHD and control groups, unless otherwise specified. Age and gender were included as covariates in all the analyses given the significant group differences on these variables. ${ }^{2}$

\footnotetext{
2 Age was not significantly correlated with bias scores in either group. Bias scores for boys and girls were not significantly different within the control sample. As estimated IQ (EIQ) did not differ between the ADHD and control groups and did not correlate with bias scores in either group, it was not entered as a covariate in the analyses.
}

\section{Results}

\section{Task performance}

The number of wins, losses, and no-outcomes experienced during the first two blocks of 100 trials was compared across the ADHD and control groups (Table 2). There was a main effect of group for the number of wins $(F(1,89)=6.27$, $p<.05)$, with the control group children experiencing significantly more wins. There were no group differences for the number of losses and no outcomes. Consequently, control group children earned more points over the two blocks of trials than children in the ADHD group $(F(1,89)=6.53$, $p<.05)$. Twenty-two children $(37.3 \%)$ in the control group, versus three children (8.8\%) in the ADHD group, reached 400 points before completing 300 trials. 


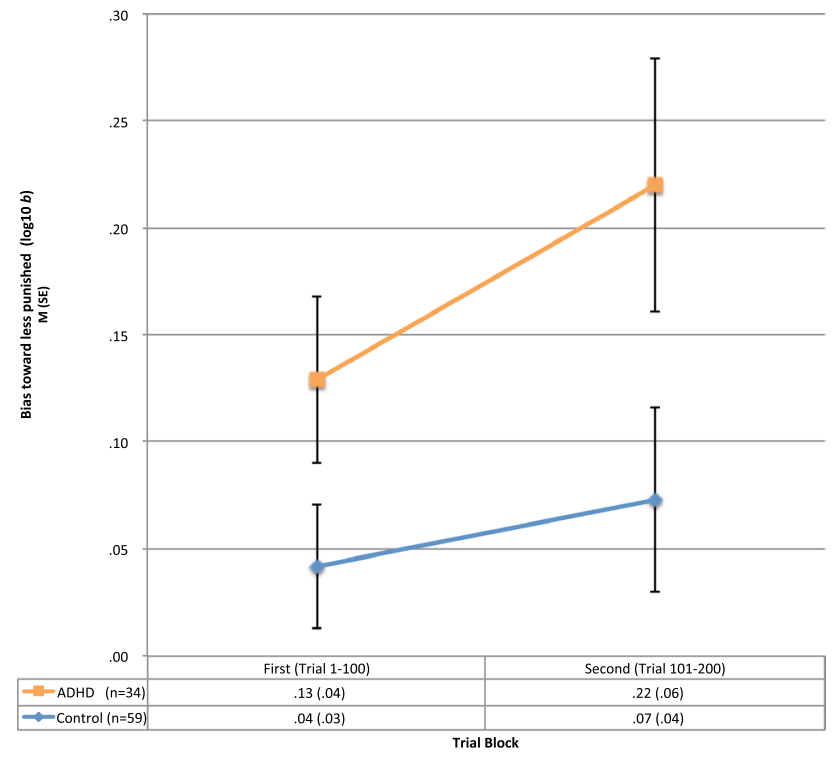

Fig. 1 Mean response bias scores and standard errors during the first and second blocks of 100 trials. Adjusted for age (110.57) and gender (1.38)

\section{Response bias}

Children in the ADHD group demonstrated a significantly larger bias toward the less frequently punished game compared to controls (group main effect: $F(1,89)=4.04$, $p<.05$ ) (Fig. 1). One-sample t-tests indicated the bias scores of the ADHD group were significantly different from zero for the first $(t(33)=2.81, p<.01)$ and second $(t(33)=3.69, p<.01)$ blocks. Those of the control group were not significantly different from zero $(t(58)=1.95$, $p<.10$ for both blocks).

\section{Frequency of stays}

\section{Across blocks}

Analysis of the logit-transformed values of the proportion of stays indicated that children with ADHD were more likely to play the same game for two consecutive trials throughout the two blocks (group main effect: $F(1,89)=6.30, p<.05$ ).

\section{Across games}

A comparison of the proportion of stays on Game 1 (more punished) versus Game 2 (less punished) yielded significant Game x Group interaction $(F(1,89)=7.13, p<.01)$ and group main $(F(1,89)=5.97, p<.05)$ effects (Fig. 2a). Post-hoc comparisons indicated children with ADHD were more likely to play the same game for two consecutive trials on the less punished than more punished alternative
$(F(1,33)=16.90, p<.001)$. This resulted in the significant group difference on the less punished game $(F(1,89)=9.83$, $p<.01$ ), with children with ADHD demonstrating a higher proportion of stays compared with controls.

\section{Across outcome types}

Examination of the proportion of stays following punishment versus non-consequential trials (across the two response alternatives) showed a trend toward main effects for outcome $(F(1,89)=2.78, p<.10)$ and group $(F(1,89)=2.89$, $p<.10$ ) (Fig. 2b). Post-hoc comparisons indicated both groups were less likely to stay after a punished than a nooutcome trial (ADHD: $F(1,33)=32.98, p<.001$, control: $F(1,58)=10.89, p<.01)$. One-way ANOVAs indicated no significant group difference on the logit-transformed proportion of stays following a punished or rewarded outcome, while children with ADHD were more likely than controls to stay on the same game following a non-consequential trial $(F(1,89)=5.59, p<.05)$.

\section{Response time}

\section{Across blocks}

Median response times declined significantly over the two blocks for both groups (block main effect: $F(1,89)=5.66$, $p<.05$ ), children responded more quickly with increased exposure to the task. A main effect of group was also observed $(F(1,89)=4.90, p<.05)$; children with ADHD responded more quickly than the controls during the first block of 100 trials $(F(1,89)=7.49, p<.01)$.

\section{Across games}

A comparison of response times for Game 1 (more punished) versus Game 2 (less punished) yielded a significant Game x Group interaction $(F(1,89)=11.21, p<.01)$, together with Game $(F(1,89)=4.44, p<.05)$ and Group $(F(1,89)=4.65, p<.05)$ main effects (Fig. $2 c)$. Children with ADHD responded more quickly on the less punished than the more punished game $(F(1,33)=13.39, p<.01)$. The median response times of the two groups were similar on the more punished alternative, while the ADHD group was significantly faster than the control group on the less punished alternative $(F(1,89)=11.78, p<.01)$.

\section{Across outcome types}

Examination of median response times after each outcome type showed a significant Outcome $\mathrm{x}$ Group interaction $(F(1.15,102.43)=7.11, p<.01$, Greenhouse-Geisser correction) (Fig. 2d). The slowest response time was observed 


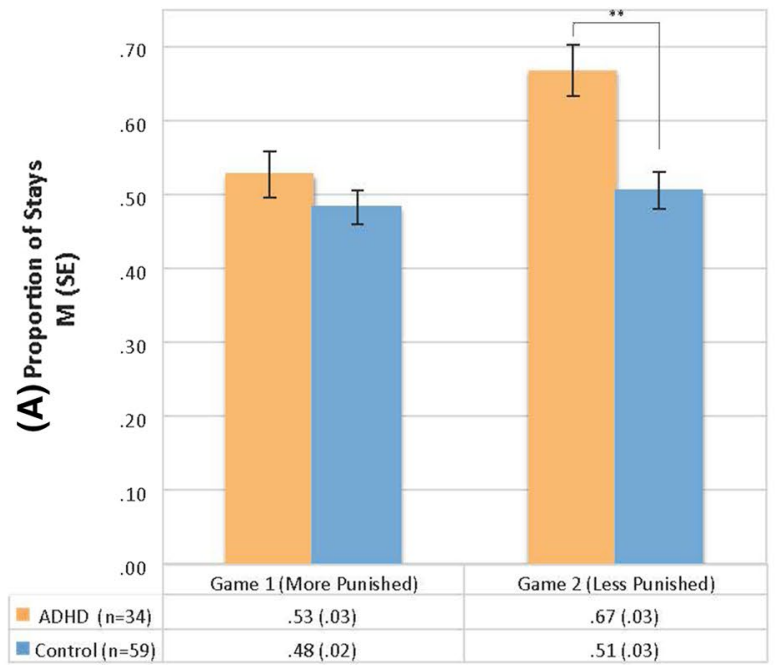

Response Alternative

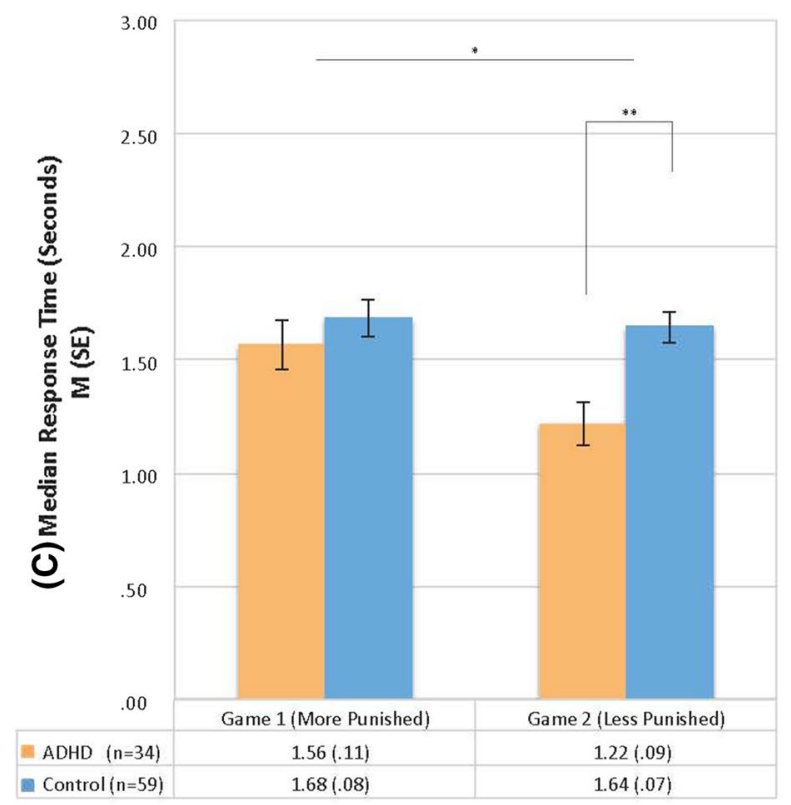

Response Alternative

Fig. 2 Means and standard errors for proportion of stays on the a two games and $\mathbf{b}$ following each trial type (significance test on the logit-transformed values), and for median response time on the c

on trials after rewards, followed by punishments and nooutcomes for both the ADHD $(F(1.06,34.98)=11.07$, $p<.01$, Greenhouse-Geisser correction) and control $(F(1.94,112.59)=20.70, p<.001$, Greenhouse-Geisser correction) groups. Post-hoc comparisons indicated the ADHD group children were significantly faster than controls after non-consequential trials $(F(1,89)=10.37$, $p<.01)$. The ADHD group was slower than the control group following rewarded and punished trials, but these differences were not significant.
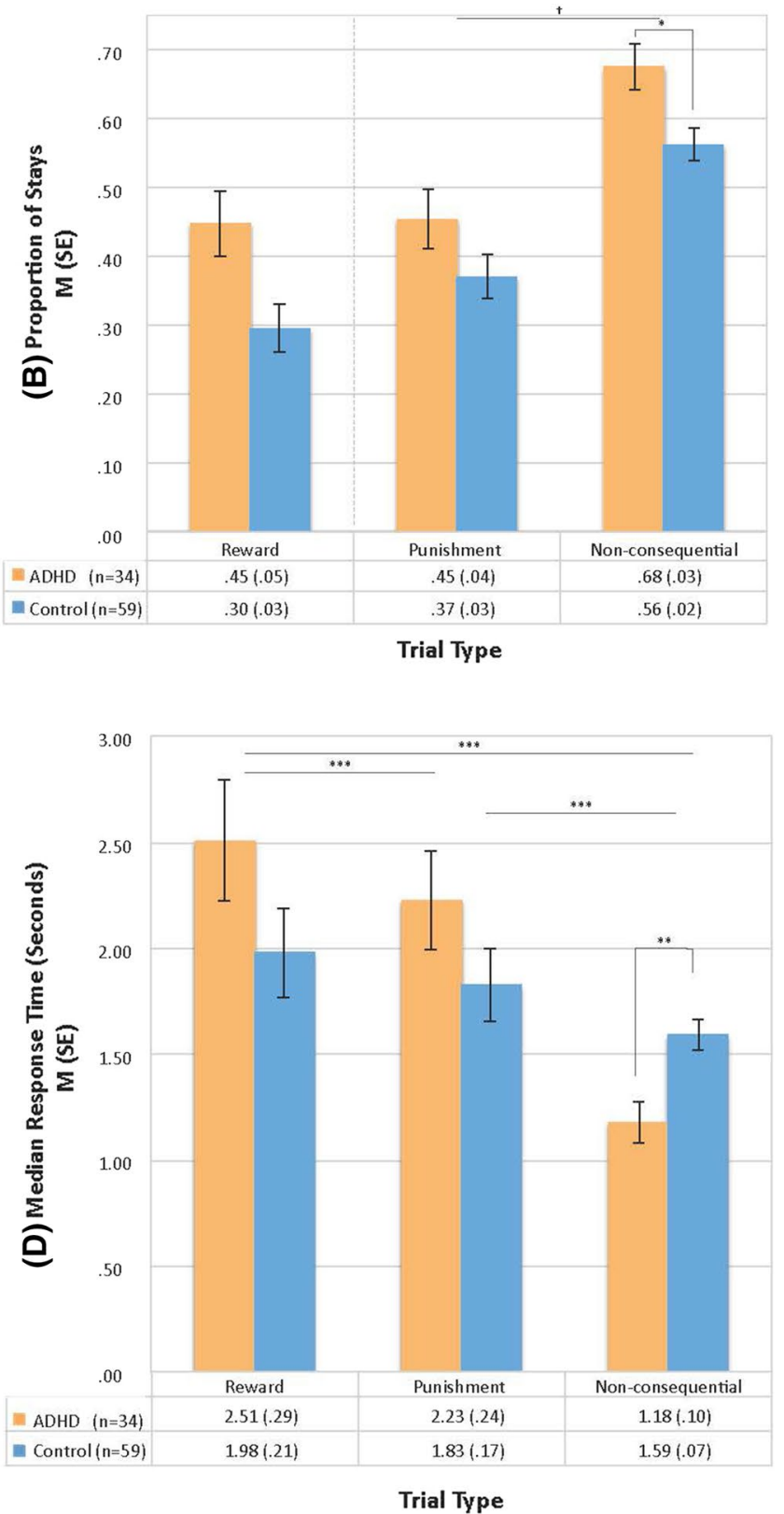

two games and $\mathbf{d}$ following each trial type for the ADHD and control groups. Adjusted for age (110.57) and gender (1.38). ${ }^{\dagger} p<.1 .0$, $* p<.05, * * p<.01, * * * p<.001$

\section{Discussion}

This study assessed the effects of unequal frequency of punishment on the response allocation of Japanese children with and without ADHD, using a concurrent operant procedure. Children with ADHD showed greater behavioral sensitivity to punishment than their typically developing peers, evidenced across a range of outcome measures. 
Children with ADHD showed a clear preference for playing the game associated with the lower frequency of punishment. Their bias scores were appreciably different from zero and significantly higher than controls across both blocks of trials. Typically developing children allocated their responses more evenly across the two games, their bias scores only marginally different from zero. These results are consistent with earlier studies showing the behavior of children with ADHD is influenced by punishment and offer further evidence that they are more sensitive to the effects of punishment than their typically developing peers (Furukawa et al. 2017).

Group differences in "stay" behavior were also found. In the controls, the rate of playing the same game on two consecutive trials was similar across the alternatives. Children with ADHD stayed less on the more punished alternative, playing the less punished alternative on two consecutive trials more often than controls. While repeating the same action is adaptive in some circumstances, i.e., the first response after a game switch was never rewarded, staying too long on the "safer" game reduced opportunities to win points. Control group children experienced more wins and accumulated more points faster than those with ADHD.

A similar pattern was seen for response speed. Children with ADHD responded more slowly on the more punished alternative, suggesting a hesitancy to engage with this game. Both groups of children slowed their responses immediately after punishments and rewards. This effect was more pronounced for children with ADHD. These findings suggest the response times of children with ADHD are impacted more by motivationally significant events than those of controls.

We employed the same experimental paradigm as Furukawa et al. (2017), allowing direct comparison of the results of the two studies. In both, the behavior of children with ADHD was more sensitive to punishment than their typically developing peers. In Furukawa et al. (2017), group differences in response bias emerged with increased time on task, leading the authors to suggest the effects of punishment are cumulative in children with ADHD. In the current study, group differences were evident earlier in the task, an effect driven by the low bias scores of the control group children. Children with ADHD in both studies evidenced similar responses to punishment, i.e., comparable mean bias scores that increased from the first to the second block of trials. The similarity in the performance of children with ADHD in the current study and that of Furukawa et al.(2017), suggests increased behavioral control by punishment may be a common characteristic of children with ADHD, irrespective of culture or country of origin ${ }^{3}$.

\footnotetext{
${ }^{3}$ Direct comparison of the Japanese ADHD group data in the current study to the English-speaking ADHD group data from the previous study (Furukawa et al. 2017) shows no significant difference in their bias scores. Details are available in Supplemental Results, including comparisons of control group data.
}

Altered neurobiological processes may underlie such increased sensitivity to punishment in ADHD; however, possible causal mechanisms have received little attention to date. Learning history, i.e., increased prior experience of punishment, may play a role. Children with ADHD engage in higher rates of inappropriate behavior in some situations; as a result, they are likely to receive more negative feedback than their peers (Seipp and Johnston 2005; Triguero Veloz Teixeira et al. 2015). In addition to increased sensitivity to punishment, poorer tracking of rewards may have also contributed to less advantageous response allocation in the ADHD group (Pollak et al. 2016). Executive functioning deficits, including working memory, are documented in children with ADHD (Willcutt et al. 2005).

The current findings are consistent with previous reports indicating the behavior and performance of children with ADHD are modified by response cost and negative feedback (e.g., Worland 1976; Rosén et al. 1984; Carlson and Tamm 2000; Drechsler et al. 2010). In contrast, increased risktaking has been reported and interpreted as reduced punishment sensitivity in ADHD (see Groen et al. 2013). In these paradigms, predominantly gambling tasks, greater risks are often accompanied by better payoffs. The current study used a simple operant paradigm, in part to reduce confounding influences of decision making or task difficulty. Multi-modal negative consequences, used to increase the outcome saliency and ecological validity, may also have influenced the study results. In the current paradigm, the negative outcome included a loss of points (response cost) and the sound of laughter (positive [social] punishment). In everyday life, response cost (e.g., loss of privileges, time out) is often accompanied by positive punishment (e.g., scolding by a parent, humiliation). How the children with ADHD would have responded to response cost alone is an open question.

Typically developing Japanese children demonstrated very little response bias during the task. This did not arise from a lack of awareness of punishment. They responded more slowly and were less likely to play the same game after punished than non-consequential trials. Thus, while these children were sensitive to individual occurrences of punishment, their overall response allocation was influenced less by the frequency of punishment. These data argue against simple habituation, which would predict reduced immediate (response time, stays) and overall (bias) effects of punishment. For the Japanese control group children, having initially distributed their responses more evenly across the two games, they experienced significantly more wins and accumulated more points than the children with ADHD. This higher rate of reinforcement may have helped maintain their more even response distribution across the two games, resulting in a lower bias. Interestingly, control children who completed all 300 trials evidenced an increase in their bias toward the less punished alternative during the third block 
of 100 trials. This is consistent with the other evidence that typically developing Japanese children are not insensitive to punishment.

Cultural expectations that one should persist, despite adversity, referred to as gaman in Japanese (Hirasuna 2005), may help explain these children's continued responding on the game that punished them more often. Gaman is to suffer and endure the unbearable with patience and dignity and is considered as a virtue in Japan. Children are often taught to exercise gaman when presented with pain, failure, loss, criticism, etc., for better eventual outcomes for oneself and/ or others. Control children in the current study kept going back to the more punished response alternative, even though they shifted away from that game immediately after punishment. This ability to return to the more punishing alternative may be encouraged by previous experiences of persisting through adversity.

In recruiting children in a country where the recognition of ADHD has a shorter history than in many Western countries, care was taken to ensure accuracy of group membership through detailed data collection and conservative application of diagnostic criteria. Age and gender differences were controlled in the analyses. Sample sizes are moderate only, because of this we did not carry out separate analyses for ADHD subtypes, comorbidity, or those completing all 300 trials versus those reaching 400 points earlier ${ }^{4}$. Given the restricted range of ADHD symptoms within each group, the analyses focused on ADHD versus control group differences. Data from children with significant cognitive and language impairments were excluded. The rate of comorbid Asperger's disorder symptoms is high and ODD low. In Japan, the availability of psychological services is limited, with elevated stigma for help seeking (Ando et al. 2013; Miyasaka et al. 2018). Families of children with severe neurodevelopmental impairments may have been more likely to volunteer for research offering comprehensive assessments. However, the high rate of co-occurring ADHD and pervasive developmental disorder (PDD) is consistent with Japanese patient profiles (Yoshida and Uchiyama 2004; Takanashi et al. 2014). While the rate of comorbid PDD raises questions about the specificity of the current findings, the response style of the Japanese children with ADHD is highly consistent with the larger sample from

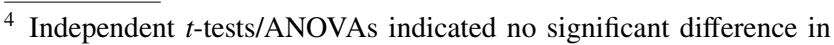
bias scores between ADHD subtypes, or those with and without any comorbid condition, or ASD/ODD comorbidity specifically, within the ADHD group, nor between the two genders, and those completing 300 trials versus those reaching 400 points in fewer trials within the control group. Independent $t$-tests and a Chi-square test indicated no significant difference in the estimated IQ, age or gender of those completing 300 trials versus fewer trials within the control group.
}

New Zealand and North America reported by Furukawa et al. (2017). This increases our confidence in the findings, despite sample limitations. Nonetheless, questions remain whether increased punishment sensitivity is unique to ADHD, shared across disorders, and/or relates to functional impairments.

Clinically, the current findings argue for additional caution in the use of punishment in the behavioral management of children with ADHD. Additional studies examining the effects of response cost versus positive punishment will help inform best practices. In this study, the children's avoidance of punishment led to poorer, rather than better, task performance, i.e., accumulation of fewer points. For children with ADHD, praise and positive attention for appropriate behavior should be emphasized. Where punishment is judged necessary, its behavioral and emotional impact should be closely monitored. This may require additional vigilance in cultures emphasizing uniformity over individualization of behavior management practices and correction of behavior through negative feedback (Markus and Kitayama 1989; Heine et al. 2001). Furthermore, the lower behavioral sensitivity of typically developing Japanese children to punishment may create a negative halo effect for children with ADHD. Their increased sensitivity to punishment being perceived as more deviant and reflecting a lack of endurance, a trait that is highly valued in Japanese culture (Berrien 1966; Hirasuna 2005).

\section{Conclusion}

Japanese children with ADHD showed increased behavioral sensitivity to the effects of punishment, offering further support for altered motivational processes contributing to symptoms of ADHD across cultures. The differences in the performance of the typically developing children in this study, to those reported previously, highlight the importance of culturally appropriate comparison groups in experimental research and clinical practice.

Cultural expectations for children's behavior vary, as do sanctions for failure to meet such expectations. This may result in differences in the degree of functional impairment experienced by children with ADHD in different cultures, despite their similar behavioral responses to punishment. The challenge may lie in persuading families, teachers, and service providers of the universality of increased sensitivity to punishment in ADHD and the need for caution in its use with this population.

Acknowledgements This research was supported by internal subsidy funding from the OIST Graduate University, Okinawa, Japan. We would like to thank all the children, families, and teachers who took part in the study. 
Availability of data and materials The datasets used and/or analyzed during the current study are available from the corresponding author on reasonable request.

\section{Compliance with ethical standards}

Conflicts of interest Shizuka Shimabukuro has received speaker fees from Shire. Other authors report no potential conflicts of interest.

Ethics approval and consent to participate Ethical approval for the study was obtained from the Okinawa Institute of Science and Technology Graduate University Human Subjects Research Review Committee. Participating parents, teachers, and children were volunteers and provided written informed consent.

Consent for publication Not applicable.

Open Access This article is distributed under the terms of the Creative Commons Attribution 4.0 International License (http://creativeco mmons.org/licenses/by/4.0/), which permits unrestricted use, distribution, and reproduction in any medium, provided you give appropriate credit to the original author(s) and the source, provide a link to the Creative Commons license, and indicate if changes were made.

\section{References}

Alsop B, Furukawa E, Sowerby P et al (2016) Behavioral sensitivity to changing reinforcement contingencies in attention-deficit hyperactivity disorder. J Child Psychol Psychiatry 57:947-956

Ando S, Yamaguchi S, Aoki Y, Thornicroft G (2013) Review of mental-health-related stigma in Japan. Psychiatry Clin Neurosci $67: 471-482$

Badger K, Anderson L, Kagan RJ (2008) Attention deficit-hyperactivity disorder in children with burn injuries. J Burn Care Res 29:724-729

Baum WM (1981) Optimization and the matching law as accounts of instrumental behavior. J Exp Anal Behav 36:387-403

Berrien FK (1966) Japanese values and the democratic process. J Soc Psychol 68:129-138

Borrero CSW, Vollmer TR, Borrero JC et al (2010) Concurrent reinforcement schedules for problem behavior and appropriate behavior: experimental applications of the matching law. J Exp Anal Behav 93:455-469

Carlson CL, Tamm L (2000) Responsiveness of children with attention deficit-hyperactivity disorder to reward and response cost: differential impact on performance and motivation. J Consult Clin Psychol 68:73-83

Carlson CL, Mann M, Alexander DK (2000) Effects of reward and response cost on the performance and motivation of children with ADHD. Cognit Ther Res 24:87-98

Critchfield TS, Paletz EM, MacAleese KR, Newland MC (2003) Punishment in human choice: direct or competitive suppression. J Exp Anal Behav 80:1-27

Crone EA, Jennings JR, van der Molen MW (2003) Sensitivity to interference and response contingencies in attention-deficit/hyperactivity disorder. J Child Psychol Psychiatry 44:214-226

Drechsler R, Rizzo P, Steinhausen H-C (2010) The impact of instruction and response cost on the modulation of response-style in children with ADHD. Behav Brain Funct 6:31
Flory K, Molina BSG, Pelham WE Jr et al (2006) Childhood ADHD predicts risky sexual behavior in young adulthood. J Clin Child Adolesc Psychol 35:571-577

Furukawa E, Alsop B, Sowerby P et al (2017) Evidence for increased behavioral control by punishment in children with attention-deficit hyperactivity disorder. J Child Psychol Psychiatry 58:248-257

Garzon DL, Huang H, Todd RD (2008) Do attention deficit/hyperactivity disorder and oppositional defiant disorder influence preschool unintentional injury risk? Arch Psychiatr Nurs 22:288-296

Groen Y, Gaastra GF, Lewis-Evans B, Tucha O (2013) Risky behavior in gambling tasks in individuals with ADHD-a systematic literature review. PLoS ONE 8:e74909

Heine SJ, Lehman DR, Markus HR, Kitayama S (1999) Is there a universal need for positive self-regard. Psychol Rev 106:766-794

Heine SJ, Lehman DR, Ide E et al (2001) Divergent consequences of success and failure in Japan and North America: an investigation of self-improving motivations and malleable selves. J Pers Soc Psychol 81:599-615

Hirasuna D (2005) The art of Gaman: arts and crafts from the Japanese American internment camps 1942-1946. Ten Speed Press, Berkeley

Humphreys KL, Tottenham N, Lee SS (2018) Risky decision-making in children with and without ADHD: a prospective study. Child Neuropsychol 24:261-276

Iaboni F, Douglas VI, Baker AG (1995) Effects of reward and response costs on inhibition in ADHD children. J Abnorm Psychol 104:232

Inoue Y, Ito K, Kita Y et al (2014) Psychometric properties of Japanese version of the Swanson, Nolan, and Pelham, version-IV ScaleTeacher Form: a study of school children in community samples. Brain Dev 36:700-706

Itani T, Kambayashi Y, Nakada Y (2001) Standardization of the Japanese version of the child behavior checklist/4-18. Psychiatr Neurol Pediatr Jpn 41:243-252

Jean-Richard-Dit-Bressel P, Killcross S, McNally GP (2018) Behavioral and neurobiological mechanisms of punishment: implications for psychiatric disorders. Neuropsychopharmacology 43:1639-1650

Kawauchi Y, Kihara N, Setoya K et al (2011) Standardization of the Japanese version of Child Behavior Checklist 2001 (CBCL/6-18). Psychiatr Neurol Pediatr Jpn 51:143-155

Kennedy M, Kreppner J, Knights N et al (2016) Early severe institutional deprivation is associated with a persistent variant of adult attention-deficit/hyperactivity disorder: clinical presentation, developmental continuities and life circumstances in the English and Romanian Adoptees study. J Child Psychol Psychiatry 57:1113-1125

Kitayama S, Markus HR, Matsumoto H, Norasakkunkit V (1997) Individual and collective processes in the construction of the self: self-enhancement in the United States and self-criticism in Japan. J Pers Soc Psychol 72:1245-1267

Lee SS, Humphreys KL, Flory K et al (2011) Prospective association of childhood attention-deficit/hyperactivity disorder (ADHD) and substance use and abuse/dependence: a meta-analytic review. Clin Psychol Rev 31:328-341

Luman M, Oosterlaan J, Sergeant JA (2005) The impact of reinforcement contingencies on $\mathrm{AD} / \mathrm{HD}$ : a review and theoretical appraisal. Clin Psychol Rev 25:183-213

Luman M, Tripp G, Scheres A (2010) Identifying the neurobiology of altered reinforcement sensitivity in ADHD: a review and research agenda. Neurosci Biobehav Rev 34:744-754

Markus HR, Kitayama S (1989) Culture and the self: implications for cognition, emotion, and motivation. Sampson 1985:1988

Masunami T, Okazaki S, Maekawa H (2009) Decision-making patterns and sensitivity to reward and punishment in children with attention-deficit hyperactivity disorder. Int J Psychophysiol $72: 283-288$ 
Miyasaka M, Kajimura S, Nomura M (2018) Biases in understanding attention deficit hyperactivity disorder and autism spectrum disorder in Japan. Front Psychol 9:244

Miyawaki D, Suzuki F, Mamoto A et al (2003) The reliability and validity of Japanese version of the schedule for affective disorders and schizophrenia for school-age children-present and lifetime version (K-SADS-PL). The 44th Congress for Establishment and Development of Child and Psychiatry at Fukuoka International Congress Center. Jpn J Child Adolesc Psychiatry 197 (In Japanese)

Pan WX, Schmidt R, Wickens JR, Hyland BI (2005) Dopamine cells respond to predicted events during classical conditioning: evidence for eligibility traces in the reward-learning network. J Neurosci 25:6235-6242

Pollak Y, Oz A, Neventsal O et al (2016) Do adolescents with attentiondeficit/hyperactivity disorder show risk seeking? Disentangling probabilistic decision making by equalizing the favorability of alternatives. J Abnorm Psychol 125:387-398

Rapport Murphy HA, Bailey JS (1982) Ritalin vs. response cost in the control of hyperactive children: a within-subject comparison. J Appl Behav Anal 15:205-216

Rasmussen EB, Newland MC (2008) Asymmetry of reinforcement and punishment in human choice. J Exp Anal Behav 89:157-167

Rosén LA, O'Leary SG, Joyce SA et al (1984) The importance of prudent negative consequences for maintaining the appropriate behavior of hyperactive students. J Abnorm Child Psychol 12:581-604

Rothbart MK, Ahadi SA (2000) Temperament and personality: origins and outcomes. J Pers Soc Psychol 78:122-135

Rothbaum F, Pott M, Azuma H et al (2000) The development of close relationships in Japan and the United States: paths of symbiotic harmony and generative tension. Child Dev 71:1121-1142

Seipp CM, Johnston C (2005) Mother-son interactions in families of boys with attention-deficit/hyperactivity disorder with and without oppositional behavior. J Abnorm Child Psychol 33:87-98

Seymour B, Singer T, Dolan R (2007) The neurobiology of punishment. Nat Rev Neurosci 8:300-311

Stubbs DA, Pliskoff SS (1969) Concurrent responding with fixed relative rate of reinforcement. J Exp Anal Behav 12:887-895

Takanashi Y, Mashiko H, Yokokawa H et al (2014) Attention-deficit/ hyperactivity disorder in adults with high-functioning pervasive developmental disorders in Japan. Open J Psychiatry 4:372

Taylor D, Lincoln AJ, Foster SL (2010) Impaired behavior regulation under conditions of concurrent variable schedules of reinforcement in children with ADHD. J Atten Disord 13:358-368
Thompson AL, Molina BSG, Pelham W Jr, Gnagy EM (2007) Risky driving in adolescents and young adults with childhood ADHD. J Pediatr Psychol 32:745-759

Thompson MJJ, Au A, Laver-Bradbury C et al (2017) Adapting an attention-deficit hyperactivity disorder parent training intervention to different cultural contexts: the experience of implementing the New Forest Parenting Programme in China, Denmark, Hong Kong, Japan, and the United Kingdom. Psych J 6:83-97

Triguero Veloz Teixeira MC, de Freitas Marino RL, Rodrigues Carreiro LR (2015) Associations between inadequate parenting practices and behavioral problems in children and adolescents with attention deficit hyperactivity disorder. Sci World J. https://doi. org/10.1155/2015/683062

van Meel CS, Oosterlaan J, Heslenfeld DJ, Sergeant JA (2005) Telling good from bad news: ADHD differentially affects processing of positive and negative feedback during guessing. Neuropsychologia 43:1946-1954

Wechsler D, Japanese WISC-III Publication Committee (1998) Japanese version of the Wechsler intelligence scale for children-third edition. Nihon Bunka Kagaku, Tokyo

Wechsler D, Japanese WISC-IV Publication Committee (2010) Japanese version of the Wechsler intelligence scale for childrenfourth edition. Nihon Bunka Kagaku, Tokyo

Wickens JR, Reynolds JNJ, Hyland BI (2003) Neural mechanisms of reward-related motor learning. Curr Opin Neurobiol 13:685-690

Willcutt EG, Doyle AE, Nigg JT et al (2005) Validity of the executive function theory of attention-deficit/hyperactivity disorder: a metaanalytic review. Biol Psychiatry 57:1336-1346

Worland J (1976) Effects of positive and negative feedback on behavior control in hyperactive and normal boys. J Abnorm Child Psychol 4:315-326

Yoshida Y, Uchiyama T (2004) The clinical necessity for assessing Attention Deficit/Hyperactivity Disorder (AD/HD) symptoms in children with high-functioning Pervasive Developmental Disorder (PDD). Eur Child Adolesc Psychiatry 13:307-314

Publisher's Note Springer Nature remains neutral with regard to jurisdictional claims in published maps and institutional affiliations. 